\title{
Micropillar single-photon source design for simultaneous near-unity efficiency and
} indistinguishability

\author{
Wang, Bi-Ying; Denning, Emil Vosmar; Gür, Uur Meriç; Lu, Chao-Yang; Gregersen, Niels
}

\section{Published in:}

Physical Review B

Link to article, DOI:

10.1103/PhysRevB.102.125301

Publication date:

2020

Document Version

Peer reviewed version

Link back to DTU Orbit

Citation (APA):

Wang, B-Y., Denning, E. V., Gür, U. M., Lu, C-Y., \& Gregersen, N. (2020). Micropillar single-photon source design for simultaneous near-unity efficiency and indistinguishability. Physical Review B, 102(12), [125301 ]. https://doi.org/10.1103/PhysRevB.102.125301

\section{General rights}

Copyright and moral rights for the publications made accessible in the public portal are retained by the authors and/or other copyright owners and it is a condition of accessing publications that users recognise and abide by the legal requirements associated with these rights.

- Users may download and print one copy of any publication from the public portal for the purpose of private study or research.

- You may not further distribute the material or use it for any profit-making activity or commercial gain

- You may freely distribute the URL identifying the publication in the public portal 


\title{
Micropillar single-photon source design for simultaneous near-unity efficiency and indistinguishability
}

\author{
Bi-Ying Wang, ${ }^{1,2}$ Emil V. Denning, ${ }^{2}$ Uğur Meriç Gür, ${ }^{3}$ Chao-Yang Lu, ${ }^{1}$ and Niels Gregersen ${ }^{2}, *$ \\ ${ }^{1}$ Hefei National Laboratory for Physical Sciences at Microscale, \\ University of Science and Technology of China, Hefei, Anhui, 230026, China \\ ${ }^{2}$ DTU Fotonik, Department of Photonics Engineering, \\ Technical University of Denmark, Ørsteds Plads, \\ Building 343, DK-2800 Kongens Lyngby, Denmark \\ ${ }^{3}$ DTU Elektro, Department of Electrical Engineering, \\ Technical University of Denmark, Ørsteds Plads, \\ Building 348, DK-2800 Kongens Lyngby, Denmark
}

(Dated: September 16, 2020)

\begin{abstract}
We present a numerical investigation of the performance of the micropillar cavity single-photon source in terms of collection efficiency and indistinguishability of the emitted photons in the presence of non-Markovian phonon-induced decoherence. We analyze the physics governing the efficiency using a single-mode model, and we optimize efficiency $\varepsilon$ and the indistinguishability $\eta$ on an equal footing by computing $\varepsilon \eta$ as function of the micropillar design parameters. We show that $\varepsilon \eta$ is limited to $\sim 0.96$ for the ideal geometry due to an inherent trade-off between efficiency and indistinguishability. Finally, we subsequently consider the influence of realistic fabrication imperfections and Markovian pure dephasing noise on the performance.
\end{abstract}

\section{INTRODUCTION}

Within the fields of optical quantum computation ${ }^{1}$ and communication $^{2}$, a key component is the single-photon source $^{3,4}$ (SPS) capable of emitting single indistinguishable photons on demand. The figures of merit include the efficiency $\varepsilon$ defined as the number of detected photons in the collection optics per trigger as well as the indistinguishability $\eta$ of consecutively emitted photons, as measured in a Hong-Ou-Mandel experiment, and their product $\varepsilon \eta$ governs the success probability of the multi-photon interference experiment ${ }^{5,6}$. The spontaneous parametric down-conversion process ${ }^{7}$ has for a long time been the workhorse for single-photon generation within the quantum optics community. While simple to implement experimentally, its main drawback is its probabilistic nature preventing multi-photon interference experiments with more than a handful of photons.

As alternative, the semiconductor quantum dot (QD) embedded in a host material ${ }^{8}$ has emerged as a promising platform for scalable optical quantum information processing. As a two-level system, the QD allows for deterministic emission of single photons and also entangled photon pairs ${ }^{9}$ through the spontaneous emission process. However, for QDs in a bulk material the high index contrast at the semiconductor-air interface reduces the collection efficiency to a few percent. The collection can be improved by placing the QD in a structured environment ${ }^{3,4}$ directing the light towards the collection optics. Design schemes allowing for vertical emission with high efficiency include the photonic nanowire ${ }^{10-13}$ design with $\varepsilon$ of $\sim 0.7$ and the "bullseye" ${ }^{9,14-17}$ planar circular Bragg grating design with $\varepsilon$ of $\sim 0.85^{16}$. These designs feature broadband $(>50 \mathrm{~nm}$ ) spectral operation which allows for easy spectral alignment of the QD and the cavity lines. However, a drawback of the broadband approach is the presence of the phonon sideband in the photon emission spectrum arising due to interaction with the solid-state environment even at zero temperature. These photons are distinguishable and constitute around $10 \%$ of the total emission, and while they can be filtered out, this occurs at the price of reduced efficiency. Another drawback is the proximity of the QD to the fluctuating charge environment ${ }^{18}$ of semiconductor-air surfaces, which again compromises the indistinguishability.

The most successful SPS design so far is the microcavity pillar ${ }^{17,19-21}$ featuring a QD in a vertical $\lambda$ cavity sandwiched between two distributed Bragg reflectors (DBRs). Almost 20 years ago, Barnes et al. proposed a model ${ }^{22}$ to quantify the efficiency in terms of the Purcell factor, from which one finds that the efficiency can be improved either by enhancing the cavity $Q$ factor or by reducing the mode volume. A practical challenge for the QD-based SPS is the random spatial and spectral distribution of typical Stranski-Krastanov-grown QDs, and important technological developments were the demonstrations of in-situ lithography ${ }^{23}$ and QD imaging techniques ${ }^{15}$ allowing for deterministic QD position control as well as Stark tuning ${ }^{21}$ to ensure the spectral alignment. The micropillar SPS then relies on narrowband Purcell enhancement to ensure efficient emission into the cavity mode, where the narrow bandwidth $(\sim 0.2$ $\mathrm{nm}$ ) enables a funneling of photons into the zero-phonon line (ZPL) and suppression of the phonon sideband allowing for high indistinguishability without any filtering. Additionally, the QD is less subject to charge noise due to a larger separation between the QD and the surfaces. These assets have led to the simultaneous demonstrations ${ }^{20,21}$ of pure $\left(g^{2}(0)<0.01\right)$ single photon emission from micropillars combining near-unity $(>0.98)$ indistinguishability with extraction efficiency of $\sim 0.65$. While, for circular micropillars half of the emitted light 
is lost in standard resonant excitation cross-polarization setups $^{20,21}$ where polarization filtering is used to suppress the laser background, this loss can be avoided using more advanced techniques like spatially orthogonal excitation and collection ${ }^{24}$ or two-colour resonant ${ }^{25}$ or phonon-assisted $^{26}$ excitation.

The question now arises of how the micropillar SPS extraction efficiency can be improved while maintaining high indistinguishability. Whereas charge noise can be effectively suppressed using metal $\operatorname{contacts}^{21,27}$, phonon-induced decoherence is not avoided even at zero temperature $^{28}$ and must be taken into account in the optimization. The efficiency can be improved by increasing the mirror reflectivities leading to a larger $Q$ factor and in turn an increased Purcell factor. However, even if one disregards unavoidable fabrication imperfections limiting the maximum achievable $Q$, a fundamental obstacle is encountered arising from the interaction with the phonon bath: While the cavity improves the indistinguishability in the weak coupling regime, increasing the Purcell factor to improve the efficiency will eventually bring the QD-cavity system into the strong coupling regime, which is detrimental to the indistinguishability. Specifically, in the strong coupling system, the system undergoes vacuum Rabi oscillations ${ }^{29}$, which causes the emission spectrum to split into two hybrid polariton states. However, in the presence of phonon coupling, the phonon-induced transitions between the upper polariton state and the lower polariton state strengthen the decoherence of the system, and hence decrease the indistinguishability ${ }^{30}$. There is thus an inherent tradeoff ${ }^{31,32}$ between the achievable efficiency and indistinguishability in the presence of phonon-induced decoherence. Consequently, an optimization of the micropillar SPS design requires accurate modeling of the efficiency as well as the indistinguishability subject to phononinduced decoherence on an equal footing, and such a study, to our knowledge, is still absent in the literature.

In this work, we perform an investigation of the achievable performance of the micropillar geometry in terms of efficiency and indistinguishability. Our theoretical method is based on two steps: First, we use a Fourier modal method ${ }^{33,34}$ to rigorously calculate the efficiency allowing for direct insight into governing physics described here using a single-mode model. Subsequently, we use the calculated optical properties of the cavity as parameter inputs to a non-Markovian master equation, which is used for evaluation of the photon indistinguishability ${ }^{31,32}$. This master equation accounts for non-Markovian phonon scattering as well as dephasing from charge noise. The influence of the sidewall roughness is also included as an additional phenomenological scattering rate. For realistic parameters of dephasing and sidewall roughness, we provide design specifications for an optimized micropillar SPS with performance significantly improved compared to state-of-theart.

This article is organized as follows: We introduce the

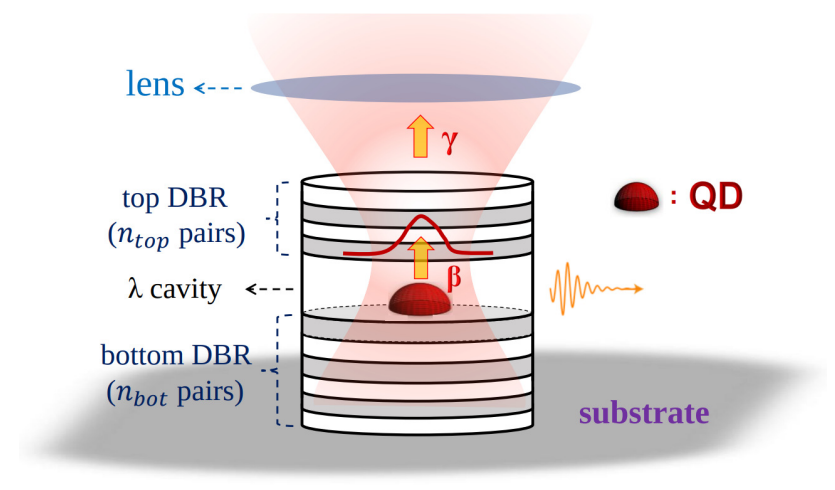

FIG. 1. Illustration of the micropillar SPS design consisting of a $\lambda$ cavity sandwiched between two DBRs. The QD, represented by the red dot, is placed at the center of the cavity. The coupling parameters $\beta$ and $\gamma$ of the single-mode model, cf. Section III A, are schematically shown.

micropillar geometry and the design recipe in Section II. The details of the optical and microscopic modeling are provided in Section III. We present our analysis of the micropillar performance and our results in Section IV, which we discuss further in Section $\mathrm{V}$ followed by a conclusion. The Appendix contains additional details of the modeling.

\section{MICROPILLAR GEOMETRY}

In this work, we investigate the micropillar cavity illustrated in Fig. 1 consisting of a QD placed in the center $\mathbf{r}_{0}$ of a $\lambda$ cavity. Light is confined vertically by the DBRs and laterally by total internal reflection. We consider an asymmetric structure with larger number $n_{\text {bot }}$ of layer pairs in the bottom DBR than the number $n_{\text {top }}$ in the top DBR to suppress leakage of light into the substrate. In the following, we fix the number of layer pairs $n_{\text {bot }}=$ 40 and study the SPS performance as function of pillar diameter $d$ and of $n_{\text {top }}$. We choose GaAs for the cavity material and $\mathrm{GaAs} / \mathrm{Al}_{0.85} \mathrm{Ga}_{0.15} \mathrm{As}$ for the DBR pairs as this choice avoids the oxidation of pure AlAs. For our design wavelength of $\lambda_{0}=895 \mathrm{~nm}$, we use a refractive index for GaAs of $3.5015(T=4 \mathrm{~K})$ and an index for $\mathrm{Al}_{0.85} \mathrm{Ga}_{0.15} \mathrm{As}$ of $2.9982^{35}$. For each pillar diameter considered, the thickness of the layers is $\operatorname{chosen}^{36}$ such that the resonance matches the design wavelength $\lambda_{0}$. Thus the cavity thickness is given by $\lambda_{0} / n_{\text {eff }}$, whereas the DBR layer thicknesses are chosen as $\lambda_{0} /\left(4 n_{\text {eff }}\right)$, where $n_{\text {eff }}$ is the diameter-dependent effective index of the fundamental mode of the section. For pillar diameters above $\sim 2.5$ $\mu \mathrm{m}$, the layer thicknesses are very close to those of the $1 \mathrm{D}$ geometry and are given by $64 \mathrm{~nm}, 75 \mathrm{~nm}$ and $256 \mathrm{~nm}$, respectively, for the $\mathrm{GaAs}$ and $\mathrm{Al}_{0.85} \mathrm{Ga}_{0.15} \mathrm{As}$ layers in the DBR and the GaAs cavity. 


\section{NUMERICAL MODEL}

Our numerical model consists of an optical simulation performed using a Fourier modal method to determine the efficiency combined with an open quantum systems master equation approach to evaluate the indistinguishability of the emitted photons in the presence of realistic decoherence effects.

\section{A. Optical modeling}

We model the QD as a classical point dipole $\mathbf{p} \delta\left(\mathbf{r}-\mathbf{r}_{0}\right)$ with in-plane orientation and harmonic time dependence with frequency $\omega$ in resonance with the cavity mode. To determine the efficiency, we compute the total emitted power $P=\frac{\omega}{2} \operatorname{Im}\left[\mathbf{p}^{*} \cdot \mathbf{E}\left(\mathbf{r}_{0}\right)\right]$ as well as the power coupled to the lens $P_{\text {Lens }}$, where we take into account an overlap with a Gaussian mode to model the coupling to a singlemode fiber, as discussed in Appendix B. The collection efficiency $\varepsilon$ is then determined as

$$
\varepsilon=I_{\mathrm{C}} \frac{P_{\text {Lens }}}{P}
$$

where $I_{\mathrm{C}}$ is a correction factor (cf. Section III C) taking into account the presence of fabrication imperfections.

While the collection efficiency is rigorously described by Eq. (1), this expression does not provide detailed understanding of the governing physics. For this reason, we also introduce a single-mode model ${ }^{4,22}$ to determine the single-mode efficiency $\varepsilon_{\mathrm{s}}$. The model describes the efficiency in terms of the spontaneous emission $\beta$ factor given by the power $P_{\mathrm{C}}$ coupled to the cavity mode over the total emitted power $P=P_{\mathrm{C}}+P_{\mathrm{R}}$, where $P_{\mathrm{R}}$ is the power coupled to all other modes. In the weak coupling regime, the $\beta$ factor can be written in terms of the Purcell factor $F_{\mathrm{p}}$ as

$$
\beta=\frac{P_{\mathrm{C}}}{P_{\mathrm{C}}+P_{\mathrm{R}}}=\frac{F_{\mathrm{p}}}{F_{\mathrm{p}}+P_{\mathrm{R}} / P_{0}},
$$

where $P_{0}$ is the power emission for a bulk medium. From Eq. (2) it is clear that $\beta$ can be improved by increasing the Purcell factor, which can be achieved by enhancing the cavity quality factor $Q$ or by reducing the cavity mode volume. The second parameter of the model is the transmission $\gamma=P_{\text {Lens, }} / P_{\mathrm{C}}$, where $P_{\text {Lens,C }}$ is the power coupled to the lens from the cavity mode alone again taking into account an overlap with a Gaussian profile. Our single mode model now describes the efficiency simply as the product $\varepsilon_{\mathrm{s}}=\beta \gamma$ as illustrated in Fig. 1 .

The calculations of $\beta, \gamma$ and $\varepsilon$ are performed using an open-geometry Fourier modal method ${ }^{33,34}$. Based on an eigenmode expansion of the optical field, the method provides direct access to the cavity mode contribution to the electric field strength $\mathbf{E}_{\mathrm{C}}(\mathbf{r})$ allowing for immediate evaluation of $P_{\mathrm{C}}$ and $P_{\text {Lens, }}$, see Appendix $A$ for further details. Finally, the spontaneous emission rate into the cavity $\Gamma_{\mathrm{C}}$ is computed from the classical power $P_{\mathrm{C}}$ using the relationship ${ }^{37} \Gamma_{\mathrm{C}} / \Gamma_{0}=P_{\mathrm{C}} / P_{0}$, where $\Gamma_{0}$ is the spontaneous emission rate in a bulk medium.

\section{B. Microscopic modeling}

We describe the QD in a solid-state environment as a two-level system, with a ground state, $|0\rangle$, and an excited state, $|e\rangle$, coupled to both photon and phonon environments. The cavity mode is described by a bosonic annihilation operator, $a$, coupled to the $\mathrm{QD}$ with a rate, $g$. The Hamiltonian describing the dynamics of the emitter and cavity is thus given by

$$
H_{0}=\hbar \omega_{\mathrm{e}}|e\rangle\langle e|+\hbar \omega_{\mathrm{c}} a^{\dagger} a+\hbar g\left(a^{\dagger} \sigma+a \sigma^{\dagger}\right),
$$

where $\omega_{\mathrm{e}}$ and $\omega_{\mathrm{c}}$ are the QD transition frequency and the cavity resonance, respectively, and $\sigma=|0\rangle\langle e|$ is the dipole operator of the QD. The cavity loss due to coupling with electromagnetic modes outside of the cavity is described by the Hamiltonian

$$
H_{c, \mathrm{EM}}=\hbar \sum_{l}\left(\omega_{A, l} A_{l}^{\dagger} A_{l}+h_{A, l} a^{\dagger} A_{l}+\text { H.c. }\right)
$$

where $A_{l}$ is the bosonic annihilation operator of the $l$ th environmental mode in the cavity loss channel, with frequency $\omega_{A, l}$ and coupling strength $h_{A, l}$ to the cavity mode. Using the cavity escape rate $\kappa_{\mathrm{c}}=\omega_{\mathrm{c}} / Q$ describing the Markovian cavity decay into all $A_{l}$ modes, we compute the QD-cavity coupling strength as $g=\frac{1}{2} \sqrt{\Gamma_{\mathrm{C}} \kappa_{\mathrm{c}}}$. Similarly, the coupling of the QD to optical radiation modes is described by

$$
H_{e, \mathrm{EM}}=\hbar \sum_{l}\left(\omega_{B, l} B_{l}^{\dagger} B_{l}+h_{B, l} \sigma^{\dagger} B_{l}+\text { H.c. }\right),
$$

where $B_{l}$ is the bosonic annihilation operator of the $l$ th environmental mode in the radiation reservoir of the $\mathrm{QD}$, with frequency $\omega_{B, l}$ and coupling strength to the emitter $h_{B, l}$. The emitter-phonon coupling is described by

$$
H_{\mathrm{P}}=\hbar \sum_{k}\left[\nu_{k} b_{k}^{\dagger} b_{k}+g_{k}|e\rangle\langle e|\left(b_{k}+b_{k}^{\dagger}\right)\right],
$$

where $b_{k}$ is the annihilation operator for the phonon mode with momentum $k$, frequency $\nu_{k}$ and coupling strength to the emitter $g_{k}$.

The total Hamiltonian is thus given by

$$
H=H_{0}+H_{c, \mathrm{EM}}+H_{e, \mathrm{EM}}+H_{\mathrm{P}} .
$$

\section{Indistinguishability}

The indistinguishability of the photon is calculated through the two-colour spectrum as ${ }^{31}$

$$
I=P_{\mathrm{S}}^{-2} \int_{-\infty}^{\infty} d \omega \int_{-\infty}^{\infty} d \nu|S(\omega, \nu)|^{2}
$$


This two-colour spectrum is defined as

$$
\left.S(\omega, \nu)=\int_{-\infty}^{\infty} d t \int_{-\infty}^{\infty} d t^{\prime}\left\langle E^{\dagger}\left(t^{\prime}\right) E(t)\right)\right\rangle e^{i\left(\omega t-\nu t^{\prime}\right)},
$$

where $E$ is the operator corresponding to the detected electric field. The frequency-integrated spectral power, $P_{\mathrm{S}}$, is defined as

$$
P_{\mathrm{S}}=\int_{-\infty}^{\infty} d \omega S(\omega, \omega)
$$

To calculate the two-colour spectrum, we use a polaron master equation, which is described in the following.

\section{Master equation}

Following previous work ${ }^{31,32,38,39}$, we make use of the Born-Markov master equation in the polaron frame. Assuming that the emitter and cavity are resonant $\left(\omega_{\mathrm{e}}=\right.$ $\left.\omega_{c}\right)$, the polaron master equation for the reduced density operator of the cavity-emitter system, $\rho$, is (in a reference frame rotating with the resonance frequency, $\omega_{\mathrm{e}}$ )

$$
\begin{aligned}
\partial_{t} \rho=-i\left[g_{\mathrm{r}} \hat{X}, \rho\right] & +\kappa_{\mathrm{c}} D(a)+\Gamma D(\sigma) \\
& +2 \gamma_{\mathrm{d}} D\left(\sigma^{\dagger} \sigma\right)+K_{\mathrm{ph}}(\rho) .
\end{aligned}
$$

Here, $g_{\mathrm{r}}=g B$ is a renormalised emitter-cavity coupling rate. The renormalisation factor is given by $B=\left\langle B_{ \pm}\right\rangle_{0}$ and $B_{ \pm}=\exp \left[ \pm \sum_{k} g_{k}^{2} / \nu_{k}\left(b_{k}^{\dagger}+b_{k}\right)\right]$, where $\langle x\rangle_{0}=\mathcal{Z}^{-1} \operatorname{Tr}\left[x e^{-\sum_{k} \hbar \nu_{k} /\left(k_{B} T\right) b_{k}^{\dagger} b_{k}}\right]$ denotes the expectation value with respect to the thermal phonon state, where $T$ is the temperature, $k_{B}$ is the Boltzmann constant and $\mathcal{Z}=\operatorname{Tr}\left[e^{-\sum_{k} \hbar \nu_{k} /\left(k_{B} T\right) b_{k}^{\dagger} b_{k}}\right]$ is the partition function. The symbol $D[x]=x \rho x^{\dagger}-\left\{x^{\dagger} x, \rho\right\} / 2$ denotes the Lindblad dissipator; $\Gamma$ represents the Markovian emission rate into the radiation modes, $B_{l}$, and $\gamma_{\mathrm{d}}$ is the pure dephasing rate. The phonon dissipator is written $\mathrm{as}^{31}$

$$
K_{\mathrm{ph}}=-g^{2}\left([\hat{X}, \hat{X} \rho] \chi_{X}+[\hat{Y}, \hat{Y} \rho] \chi_{\mathrm{Y}}+[\hat{Y}, \hat{Z} \rho] \chi_{Z}+\text { H.c. }\right) \text {, }
$$

with $\hat{X}=\sigma^{\dagger} a+\sigma a^{\dagger}, \hat{Y}=i\left(\sigma^{\dagger} a-\sigma a^{\dagger}\right)$ and $\hat{Z}=\sigma^{\dagger} \sigma-a^{\dagger} a$. The coefficients are

$$
\begin{aligned}
& \chi_{X}=\int_{0}^{\infty} d \tau \Lambda_{X X}(\tau), \\
& \chi_{Y}=\int_{0}^{\infty} d \tau \cos \left(2 g_{\mathrm{r}} \tau\right) \Lambda_{Y Y}(\tau), \\
& \chi_{Z}=-\int_{0}^{\infty} d \tau \sin \left(2 g_{\mathrm{r}} \tau\right) \Lambda_{Y Y}(\tau),
\end{aligned}
$$

with the phonon correlation functions $\Lambda_{X X}(\tau)=$ $\left\langle B_{X}(\tau) B_{X}\right\rangle=B^{2}[\sinh \varphi(\tau)-1]$ and $\Lambda_{Y Y}(\tau)=\left\langle B_{Y}(\tau) B_{Y}\right\rangle=$ $B^{2} \cosh \varphi(\tau)$ with $B_{X}=\left(B_{+}+B_{-}-2 B\right) / 2$ and $B_{Y}=$ $i\left(B_{+}-B_{-}\right)$. Here, $\varphi(\tau)$ is given by ${ }^{38}$

$$
\varphi(\tau)=\int_{0}^{\infty} d \omega \frac{J(\omega)}{\omega^{2}}\left[\operatorname{coth}\left(\frac{\hbar \omega}{2 k_{\mathrm{B}} T}\right) \cos (\omega \tau)-i \sin (\omega \tau)\right]
$$

where the phonon spectral density is $J(\nu)=$ $\sum_{k}\left|g_{k}\right|^{2} \delta\left(\nu-\nu_{k}\right)=\alpha \nu^{3} \exp \left(-\nu^{2} / \nu_{\mathrm{c}}^{2}\right)$ with $\alpha$ being exciton-phonon coupling strength and $\nu_{\mathrm{c}}=\sqrt{2} v_{\mathrm{c}} / L$ denoting the cutoff frequency ${ }^{40}$, where $v_{\mathrm{c}}$ and $L$ are the speed of sound and the size of the QD, respectively. Here, the exciton-phonon coupling strength $\alpha$ depends on the QD material and on the cutoff frequency $\nu_{\mathrm{c}}$, which itself is related to the size of the $\mathrm{QD}^{41}$. The Franck-Condon factor, $B^{2}$, can be written compactly in terms of this function as $B^{2}=e^{-\varphi(0)}$.

The master equation, Eq. (11), allows us to calculate the two-colour spectrum, $S(\omega, \nu)$. This is done by first calculating the dipole spectrum,

$$
\begin{aligned}
S_{0}(\omega, \nu)=\int_{0}^{\infty} d t \int_{0}^{\infty} & d \tau e^{i(\omega-\nu) t} e^{-i \omega \tau} \\
& \times G_{\mathrm{P}}(\tau)\left\langle\sigma^{\dagger}(t+\tau) \sigma(t)\right\rangle,
\end{aligned}
$$

where $G_{\mathrm{P}}(\tau)=B^{2} e^{\varphi(\tau)}$ is the free phonon Green's function and the two-time dipole correlation function, $\left\langle\sigma^{\dagger}(t+\tau) \sigma(t)\right\rangle$, is evaluated using the quantum regression theorem with Eq. (11). From this dipole spectrum, the detected field spectrum is calculated as ${ }^{31}$

$$
S(\omega, \nu)=G_{\mathrm{F}}(\omega, \nu) S_{0}(\omega, \nu)+G_{\mathrm{F}}^{*}(\nu, \omega) S_{0}^{*}(\nu, \omega),
$$

where

$$
G_{\mathrm{F}}(\omega, \nu)=\frac{\left(\kappa_{\mathrm{c}} / 2\right)^{2}}{\left(i \omega-\kappa_{\mathrm{c}} / 2\right)\left(-i \nu-\kappa_{\mathrm{c}} / 2\right)}
$$

is an optical two-colour Green's function connecting the dipole with the field emitted from the cavity. In the Purcell-regime, where $g \ll \kappa_{\mathrm{c}}$, the indistingusihability can be approximated by the expression

$$
I \simeq \frac{\Gamma_{\mathrm{t}}}{\Gamma_{\mathrm{t}}+2 \gamma_{\mathrm{t}}}\left[\frac{B^{2}}{B^{2}+F\left(1-B^{2}\right)}\right]^{2},
$$

where $\Gamma_{\mathrm{t}}=4 g^{2} / \kappa_{\mathrm{c}}+\Gamma$ is the total Purcell-enhanced spontaneous emission rate of the $\mathrm{QD}, \gamma_{\mathrm{t}}=\gamma_{\mathrm{d}}+$ $2 \pi\left(g_{\mathrm{r}} / \kappa_{\mathrm{c}}\right)^{2} J\left(2 g_{\mathrm{r}}\right) \operatorname{coth}\left(\hbar g_{\mathrm{r}} / k_{B} T\right)$ is a phonon-enhanced pure dephasing rate and $F$ is the fraction of photons in the phonon sideband not eliminated by the cavity ${ }^{31}$.

Through narrowband Purcell enhancement, the micropillar SPS ensures an efficient emission into the cavity mode, where the narrow bandwidth $(\sim 0.2 \mathrm{~nm})$ enables a funneling of photons into the ZPL and a suppression of the phonon sideband allowing for high indistinguishability without any filtering. To illustrate this influence, we show the spectra $S(\omega, \omega)$ for a low $\mathrm{Q}$ and a high Q micropillars in Fig. 2 both exhibiting a narrow peak and a broadband base corresponding to the ZPL and the phonon sideband, respectively. We observe that the high Q micropillar leads to a broadened ZPL as a result of the large Purcell factor and a suppression of the phonon sideband. 


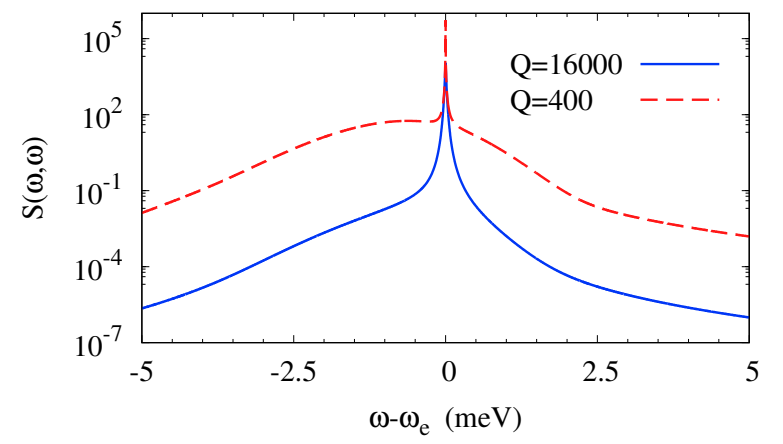

FIG. 2. Emission spectrum showing the zero-phonon line and the phonon sidebands at $T=4 \mathrm{~K}$. Dashed curve: $Q=400$ and $F_{\mathrm{p}}=2$; solid curve: $F_{\mathrm{p}}=20$ and $Q=16000$. Parameters: $\alpha=0.03 \mathrm{ps}^{-2}$ and $\hbar \nu_{\mathrm{c}}=1.4 \mathrm{meV}$ following Ref. 31 .

\section{Modeling of fabrication imperfections}

While the figures of merit of the micropillar SPS can be computed accurately using the formalisms presented above, the influence of unavoidable fabrication imperfections leading to discrepancy ${ }^{42,43}$ between the prediction of the modeling and the experimentally measured performance should be taken into account. The imperfections can be described phenomenologically by introducing additional loss channels due to sidewall roughness and material absorption ${ }^{44}$. The quality $Q$ factor is then written as $^{19,45}$

$$
\frac{1}{Q}=\frac{1}{Q_{\text {int }}}+\frac{1}{Q_{\text {edge }}}+\frac{1}{Q_{\text {abs }}}
$$

where the intrinsic $Q_{\text {int }}$ is computed for the perfect structure ${ }^{43}$, and $Q_{\text {edge }}^{-1}$ and $Q_{\text {abs }}^{-1}$ represent the contributions to loss from sidewall scattering and material absorption, respectively. Since $F_{\mathrm{p}}$ is linearly proportional to $Q$, the introduction of imperfections leads to a corrected Purcell factor given by $F_{\mathrm{p}}^{\mathrm{c}}=F_{\mathrm{p}}\left(Q / Q_{\text {int }}\right)$ and in turn a corrected $\beta$ factor $\beta^{\mathrm{c}}=F_{\mathrm{p}}^{\mathrm{c}} /\left(F_{\mathrm{p}}^{\mathrm{c}}+P_{\mathrm{R}} / P_{0}\right)$. We then model the correction $I_{\mathrm{C}}$ to the efficiency in the presence of imperfections as $I_{\mathrm{C}}=\beta^{\mathrm{c}} / \beta$. Moreover, the corrected indistinguishability is obtained by evaluating the decay rate $\kappa_{\mathrm{c}}=\omega / Q$ in Eq. (11) using the $Q$ given by Eq. (19).

We model the sidewall scattering using a phenomenological model ${ }^{44}$ discussed in Appendix D, where $\kappa$ is a phenomenological constant proportional to the sidewall scattering rate $Q_{\text {edge }}^{-1}$. The effect of material absorption loss can be modeled either using $Q_{\text {abs }}^{-1}$ in Eq. (19) or by introducing an imaginary part ${ }^{43,46}$ to the refractive index. However, the effect of material loss is typically negligible ${ }^{43,46}$ in micropillars with $Q$ factors below $\sim 10^{5}$ and is thus ignored in this work.

\section{RESULTS}

In this section, we first present calculations of the single-mode model parameters to elucidate the physics governing the collection efficiency, after which we present results from full simulations of the efficiency and indistinguishability taking into account fabrication imperfections. All calculations are performed at a numerical aperture (NA) of 0.82 for the first lens. Following Ref. 31, we use the parameters $\hbar \nu_{\mathrm{c}}=1.4 \mathrm{meV}$ and $\alpha=0.03 \mathrm{ps}^{2}$ corresponding to a typical QD of size $25 \mathrm{~nm}^{41}$.

\section{A. Analysis using the single-mode model}

We first present the cavity $Q$ factor as function of the pillar diameter $d$ and of the number of layer pairs $n_{\text {top }}$ in the top DBR in Fig. 3(a) for the ideal structure. An increase in $n_{\text {top }}$ leads to a higher reflectivity resulting in an increased cavity $Q$ factor. For large diameters, the $Q$ factor approaches the value of a planar cavity whereas for short diameters it drops due to poor modal overlap of the $\mathrm{HE}_{11}$ mode in the cavity section and the corresponding Bloch mode in the DBR sections. Oscillations are observed for the largest values of $n_{\text {top }}$ arising from coupling $36,43,47$ with higher-order Bloch modes of the DBRs. This behavior is also observed for the Purcell factor $F_{\mathrm{p}}$ illustrated in Fig. 3(b), where the reduction of the pillar diameter $d$ and in turn the mode volume additionally leads to an overall increase in $F_{\mathrm{p}}$ until the drop of the $Q$ at around $d \sim 1.2 \mu \mathrm{m}$ sets in. As expected, the increase in the Purcell factor $F_{\mathrm{p}}$ is directly reflected in the calculated spontaneous emission $\beta$ factor presented in Fig. 3(c). However, the observed oscilla-
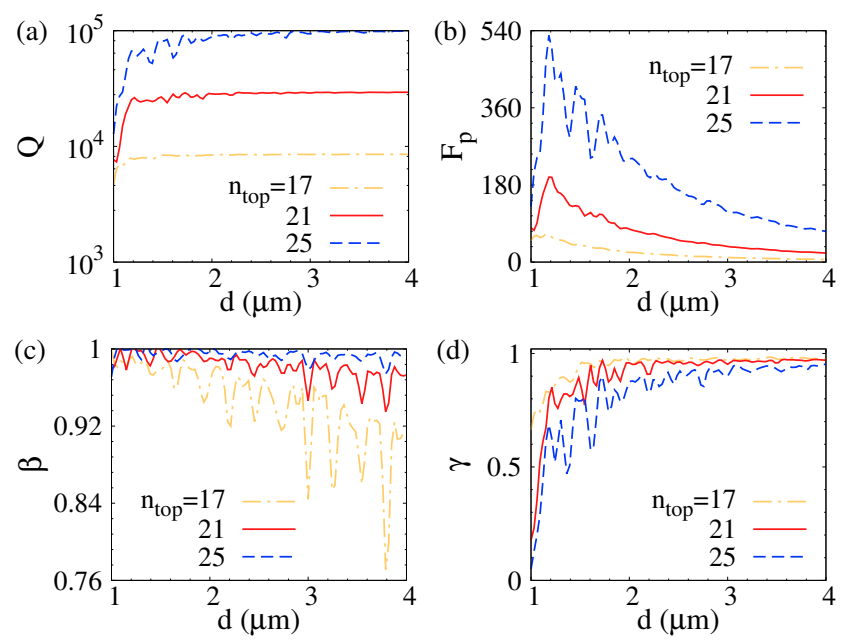

FIG. 3. Computed cavity $Q$ factor (a), Purcell factor $F_{\mathrm{p}}$ (b) and spontaneous emission $\beta$ factor (c) as well as the transmission $\gamma(\mathrm{d})$ to the lens as a function of pillar diameter $d$ for different numbers of layer pairs $n_{\text {top }}$ in the top DBR. The ideal structure with $\gamma_{\mathrm{d}}=\kappa=0$ is considered. 
tions in $\beta$ are not originating from variations in $Q$ but rather from diameter-dependent variations in the emission $P_{\mathrm{R}}$ to background modes, and they are reduced in magnitude with increasing $F_{\mathrm{p}}$ in agreement with Eq. (2). While the best coupling $\beta$ to the cavity is obtained for the diameter of $\sim 1.1 \mu \mathrm{m}$ corresponding to the maximum Purcell factor in Fig. 3(b), the transmission $\gamma$ presented in Fig. 3(d) for this $d$ is modest due to its highly divergent output beam profile ${ }^{48}$. As the diameter increases, the divergence is reduced and $\gamma$ is improved. Also, where $\beta$ is maximum for $n_{\text {top }}=25$, an increasing top mirror reflectivity leads to an increasing leakage of light into the substrate and thus a reduction of $\gamma$ for $n_{\text {top }}=25$ as shown in Appendix E.

We thus observe that there exists a trade-off between the coupling $\beta$ to the cavity and the transmission $\gamma$ to the lens. Since the efficiency is the product of the two, one may ask if the overall performance can be optimized by choosing a large diameter and by increasing the reflectivity of both DBRs while keeping $n_{\text {bot }}$ comfortably larger than $n_{\text {top }}$ to avoid leakage of light into the substrate. In the following, we will see that this is not the case.

\section{B. Analysis using the full model}

Having established our understanding of the variations of $\beta$ and $\gamma$, we now present the collection efficiency computed using the full model Eq. (1). As shown in Appendix $\mathrm{C}$, excellent agreement between the single-mode model and the full model is observed, and the variations in the efficiency can thus be fully understood from the behavior of $\beta$ and $\gamma$. The collection efficiency $\varepsilon=\beta \gamma$ is presented in Fig. 4(a) for the ideal structure without fabrication imperfections. The reduction in $\varepsilon$ for low diameters due to the drop in $\gamma$ is clearly observed, and we observe that an efficiency of $\sim 0.95$ is obtained in a broad regime for diameters above $\sim 2 \mu \mathrm{m}$ and $n_{\text {top }} \sim 21$.

The computed indistinguishability $\eta$ in the presence of phonon-induced decoherence for the otherwise ideal structure is shown in Fig. 4(b). The computed indistinguishability initially increases from 17 to 21 layer pairs as the influence of dephasing is overcome by the increase in $F_{\mathrm{p}}$ in agreement with Eq. (18). However, for $n_{\text {top }} \geq 21$ the high $Q$ factor results in significant drops in $\eta$ for decreasing pillar diameter $d$ due to the onset of the strong coupling regime $\left(4 g \gtrsim \kappa_{\mathrm{c}}\right)$ detrimental to the indistinguishability ${ }^{31}$. This drop becomes more pronounced as $n_{\text {top }}$ increases and the decay rate $\kappa_{\mathrm{c}}$ decreases.

To answer our previous question, we realize that the increase of bottom mirror $n_{\text {bot }}$ layer pairs to increase the transmission $\gamma$ for $n_{\text {top }} \geq 25$ may improve the efficiency but will simultaneously bring us even further into the regime of strong coupling detrimental to $\eta$. On the other hand, a good performance with $\eta>0.995$ is obtained in a regime at the boundary of strong coupling, in the case of $n_{\text {top }}=21$ for $d$ larger than $\sim 2 \mu m$. Overall, $\eta$ is increased towards unity by decreasing $\kappa_{\mathrm{c}}$ and by increasing

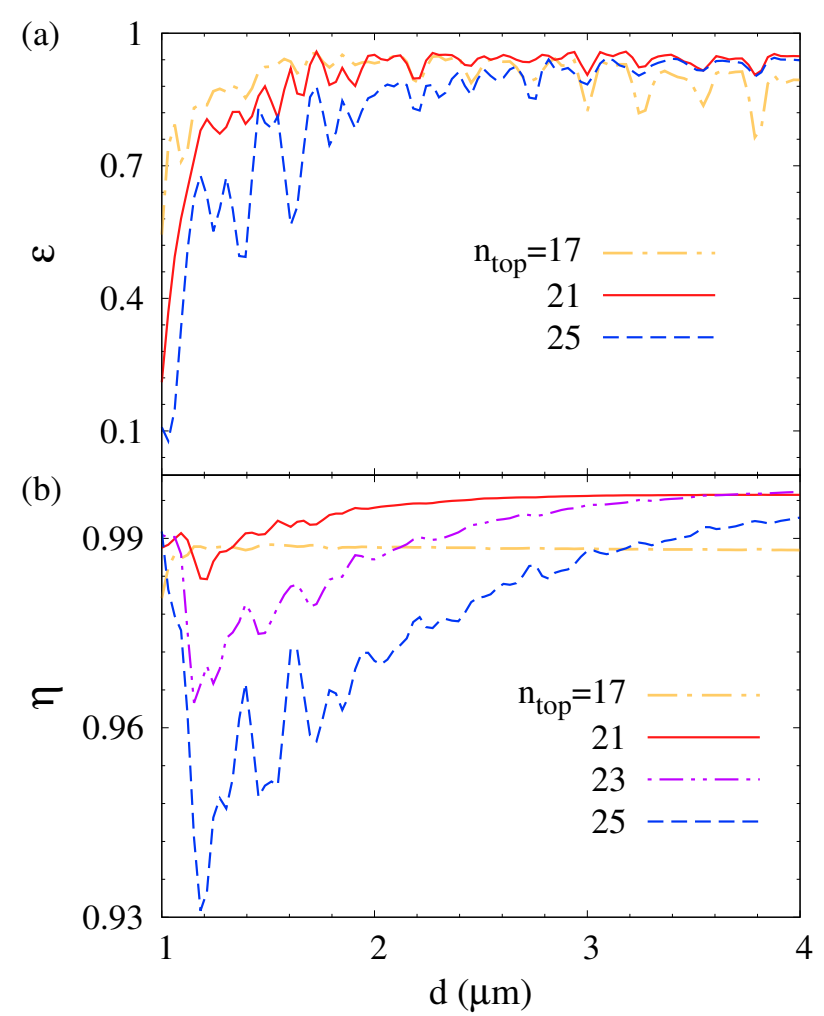

FIG. 4. Computed efficiency $\varepsilon$ (a) and indistinguishability $\eta$ (b) versus pillar diameter $d$ for different layer pairs $n_{\text {top }}$ in the top DBR for the ideal geometry with $\gamma_{\mathrm{d}}=\kappa=0$.

the diameter, which should be chosen sufficiently large to ensure weak coupling. However, as shown in Figs. 3(b,c) the increase in $d$ ultimately leads to a reduction in efficiency. An optimization of the micropillar performance in terms of $\varepsilon$ and $\eta$ is thus necessary to identify the optimal performance.

\section{Optimization}

To optimize the efficiency and indistinguishability on an equal footing, we consider as figure of merit the product $\varepsilon \eta$ presented for the ideal case without charge noise and sidewall roughness in Fig. 5(a). Here, by directly reading the maximum of $\varepsilon \eta$ in the numerical simulation, the best performance of $\varepsilon \eta \sim 0.95$ is obtained for $n_{\text {top }}=21$ at various peak $d$ positions in the interval $[2,4]$ $\mu \mathrm{m}$, where the observed variations are direct reflections of those for the $\beta$ factor in 3(b). In this regime, we have $\varepsilon \sim 0.95$ and $\eta \sim 0.997$, in good agreement with the performance optimum identified in Ref. 31.

We now introduce imperfections initially in the form of a finite dephasing rate $\hbar \gamma_{\mathrm{d}}=0.086 \mu \mathrm{eV}^{49}$ due to non-phononic Markovian decoherence mechanisms, e.g. charge noise due to an unstable charge environment ${ }^{18}$. Compared to the ideal case, the indistinguishability is reduced by the additional charge noise decoherence mech- 


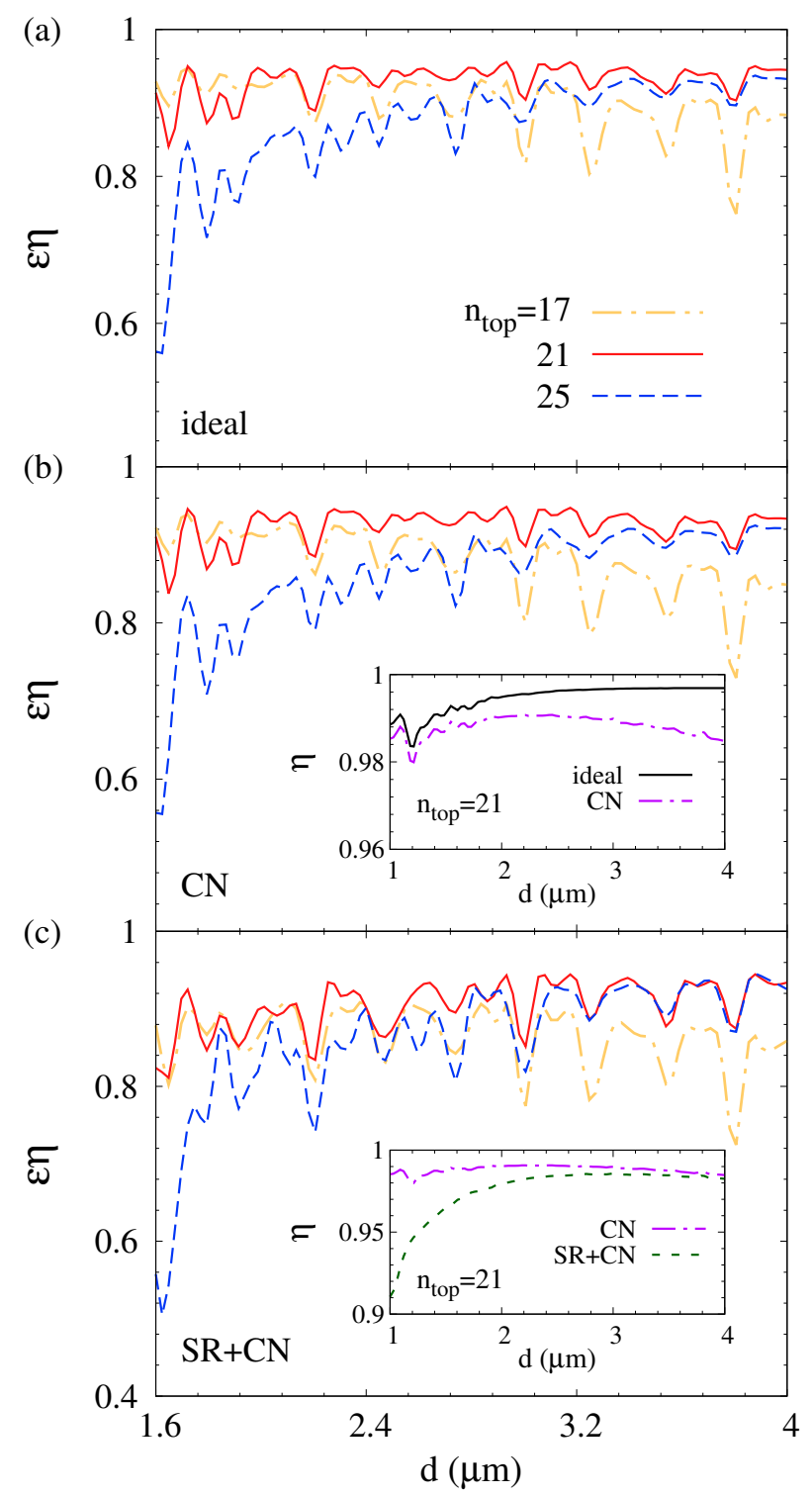

FIG. 5. The computed figure of merit $\varepsilon \eta$ as function of pillar diameter $d$ for different layer pairs $n_{\text {top }}$ in the top DBR for (a) the ideal case, (b) in the presence of charge noise $(\mathrm{CN})$ only and (c) with both charge noise and sidewall roughness (SR) present. The insets compare the indistinguishability in the three cases for $n_{\text {top }}=21$. Parameters: $\hbar \gamma_{\mathrm{d}}=0.086 \mu \mathrm{eV}$; $\kappa=0.2 \mu \mathrm{m}$.

anism. As shown in the inset of Fig. 5(b), the reduction in $\eta$ increases with $d$ due to the corresponding reduction in the Purcell factor in agreement with Eq. (18). The resulting figure of merit $\varepsilon \eta$ is shown in Fig. 5(b), where $\varepsilon \eta$ is reduced to $\sim 0.94$ for $n_{\text {top }}=21$ and $d \in[2,3.25]$ $\mu \mathrm{m}$, where $\varepsilon \sim 0.95$ and $\eta \sim 0.99$.

As a second element of imperfection, we now also take sidewall roughness into account by introducing a relatively large phenomenological constant $\kappa=0.2 \mu \mathrm{m}^{19}$, and the results are plotted in Fig. 5(c). We observe that a maximum $\varepsilon \eta$ of $\sim 0.93$ is obtained for $n_{\text {top }}=21$ and $d \in$
$[3.05,3.9] \mu \mathrm{m}$, where $\varepsilon \sim 0.945$ and $\eta \sim 0.98$. Whereas the charge noise only influenced the indistinguishability, the sidewall imperfections are detrimental to both $\varepsilon$ and $\eta$ through the reductions in $I_{\mathrm{C}}$ and $F_{\mathrm{p}}$ due to the decrease in $Q$. The reduction of $\eta$ is shown in the inset of Fig. 5(c), where the impact of the increase in sidewall scattering $Q_{\text {edge }}^{-1}$ with decreasing diameter is directly observed.

While charge noise can be overcome by increasing $F_{\mathrm{p}}$, this ultimately will lower $\eta$ due to the onset of strong coupling. Similarly, while the effect of sidewall imperfections is reduced for increasing pillar diameter, this also leads to a decrease in $F_{\mathrm{p}}$ and in turn the efficiency. The detrimental effects of charge noise and sidewall imperfections thus cannot be completely overcome in the design process alone, and they should be addressed in the fabrication, e.g. by introducing metal contacts ${ }^{21}$ for charge stabilization and using a reduced $\mathrm{Al}$ content in the DBRs to avoid oxidation.

\section{DISCUSSION}

While our simulations identify geometrical parameters leading to predicted performance significantly beyond state-of-the-art, the exact performance obtained experimentally will depend on unknown experimental parameters. Even though the exciton-phonon coupling strength $\alpha$ and the cutoff frequency $\nu_{\mathrm{c}}$ are QD-dependent, previous investigations have revealed that the optimum cavity linewidth for maximum indistinguishability is independent of the QD size in the $10-40 \mathrm{~nm}$ range ${ }^{41}$. Futhermore, the magnitude of the Markovian noise term as well as the unavoidable fabrication imperfections influence the performance. Nevertheless, our study reveals that the diameter range from 2.5 to $4 \mu \mathrm{m}$ for the $n_{\text {top }}=21$ configuration leads to the optimal micropillar SPS performance, and in our study, this conclusion is again not altered by the presence of realistic pure dephasing or fabrication imperfections, which instead simply lead to an overall degradation of the performance by a few percent. In the ideal performance regime, $\varepsilon \eta$ in Fig. 5 display variations of $\sim 5 \%$ as function of $d$. Interestingly, these variations are not caused by the well-established diameter-dependent variations ${ }^{43,47}$ in $Q$ due to coupling with higher-order Bloch modes, and an adiabatic cavity $\operatorname{design}^{50}$ will thus not eliminate the variations. Rather, Figs. 3(c,d) show that the $\varepsilon \eta$ variations appear due to strong variations with $d$ of the background emission rate $P_{\mathrm{R}} / P_{0}$, which is often simply assumed equal to unity. A better physical understanding of the background emission rate in micropillars is thus desirable but beyond this work.

However, even with such understanding, the micropillar geometry does not provide a mechanism to completely suppress the background emission. The consequence is that the trade-off between efficiency and indistinguishability is unavoidable, and a figure of merit $\varepsilon \eta$ for the mi- 
cropillar above $\sim 0.96$ is out of reach. While the micropillar geometry presently remains the champion device for efficient generation of single indistinguishable photons, this limitation motivates further investigation of alternative design strategies such as the photonic nanowire SPS $^{51,52}$, where the background emission can be controlled using dielectric screening effects ${ }^{53}$.

Whereas we predict an $\varepsilon \eta \sim 0.96$, experimentally demonstrated figures of merit ${ }^{20,21}$ are significantly below this value. This discrepancy is partly due to the cross-polarization setup in resonant excitation used in these works, reducing the efficiency by a factor of 2 . This reduction is not a fundamental limitation and can be avoided using e.g. phonon-assisted excitation ${ }^{26}$ for the rotationally symmetric micropillars considered in this work or by the introduction of an elliptical crosssection ${ }^{17}$. Even so, these methods will not overcome the inherent trade-off between efficiency and indistinguishability of the micropillar SPS. Nevertheless, an optimization of also the elliptical micropillar design in terms of efficiency and indistinguishable polarized photon emission remains of interest but is beyond this work.

\section{CONCLUSION}

In summary, by applying a Fourier modal method and a polaron master equation approach, we have numerically calculated the efficiency and indistinguishability of the microcavity pillar single-photon source. To elucidate the mechanism governing the efficiency, we have presented a single-mode model, which fully captures the physics in terms of the initial spontaneous emission to the cavity mode and the subsequent transmission to the first lens. By varying the number of DBR layer pairs and the diameter, we have observed the onset of the trade-off between efficiency and indistinguishability predicted previously, and we have identified design parameters leading to a maximum figure of merit $\varepsilon \eta$ of the product of the efficiency $\varepsilon$ and the indistinguishability $\eta$ of $\sim 0.96$ for the ideal geometry. In addition, we have investigated the influence of pure dephasing as well as sidewall scattering on the performance. Using realistic values for these mechanisms, we have shown that, while the performance is degraded by imperfections, the optimum design parameters are unchanged.

\section{ACKNOWLEDGEMENT}

The authors thank Jean-Michel Gérard for fruitful discussions. EVD and NG were supported by the Independent Research Fund Denmark, grant No. DFF-418100416 and DFF-9041-00046B. BW acknowledges support from the China Scholarship Council. This work was supported by the Danish National Research Foundation through NanoPhoton - Center for Nanophotonics, grant no. DNRF147.

\section{APPENDIX}

In the Fourier modal method, the geometry is divided into layers uniform along a propagation $z$ axis, and the electric field in each layer is expanded upon the forward and backwards propagating eigenmodes $\mathbf{e}_{\mathrm{j}}^{ \pm}\left(\mathbf{r}_{\perp}\right) e^{ \pm i \beta_{\mathrm{j}} z}$ that are solutions to the wave equation for the layer assuming uniformity along $z$. The fields on each side of the interface between layers $q$ and $q+1$ are related by interface reflection $\overline{\bar{R}}_{\mathrm{q}, \mathrm{q}+1}$ and transmission $\overline{\bar{T}}_{\mathrm{q}, \mathrm{q}+1}$ matrices determined from the boundary conditions at the interface, and the propagation through a layer of length $L$ is described by a propagation $P_{L}$ matrix with elements $e^{i \beta_{\mathrm{j}} L}$ in its diagonal. The total reflection matrix $\overline{\bar{R}}_{\mathrm{t}}\left(\overline{\bar{R}}_{\mathrm{b}}\right)$ for the top (bottom) DBR mirror is obtained from interface and propagation matrices in an iterative manner using the $\mathrm{S}$ matrix formalism. The total field at the position $z_{0}+\delta z$ just above the QD $(\delta z \rightarrow 0)$ is given by

$$
\mathbf{E}\left(\mathbf{r}_{\perp}, z_{0}+\delta z\right)=\sum_{\mathbf{j}}\left[a_{\mathbf{j}} \mathbf{e}_{\mathbf{j}}^{+}\left(\mathbf{r}_{\perp}\right)+b_{\mathbf{j}} \mathbf{e}_{\mathbf{j}}^{-}\left(\mathbf{r}_{\perp}\right)\right],
$$

where the field amplitude vectors $\bar{a}$ and $\bar{b}$ represent the field expansion just above the QD. The forward propagating field vector $\bar{a}$ is computed from the point dipole $\mathbf{p} \delta\left(\mathbf{r}-\mathbf{r}_{0}\right)$ using the reciprocity theorem and taking into account multiple round trips inside the cavity ${ }^{34}$. The backwards propagating field is obtained as $\bar{b}=$ $\overline{\bar{P}}_{L / 2} \overline{\bar{R}}_{\mathrm{t}} \overline{\bar{P}}_{L / 2} \bar{a}$.

\section{A. Cavity contribution to the field}

To determine the contribution $\mathbf{E}_{\mathrm{C}}$ from the cavity mode to the total field given in Eq. (20), we define the eigenvectors $\bar{c}_{\mathrm{k}}^{+}$of the roundtrip matrix $\overline{\bar{R}}^{+}=$ $\overline{\bar{P}}_{L / 2} \overline{\bar{R}}_{\mathrm{b}} \overline{\bar{P}}_{L} \overline{\bar{R}}_{\mathrm{t}} \overline{\bar{P}}_{L / 2}$ as solutions to the eigenproblem

$$
\overline{\bar{R}}^{+} \bar{c}_{\mathrm{k}}^{+}=\lambda_{\mathrm{k}}^{+} \bar{c}_{\mathrm{k}}^{+}
$$

where $\lambda_{\mathrm{k}}^{+}$are eigenvalues with $\left|\lambda_{\mathrm{k}}^{+}\right|<1$. For normalized eigenvectors, the cavity roundtrip eigenvector $\bar{c}_{\mathrm{M}}^{+}$corresponding to the fundamental cavity mode is easily identified as the one with the first element having a maximum value such that $\left|c_{1 \mathrm{M}}^{+}\right| \geq\left|c_{1 \mathrm{k}}^{+}\right|$. For the forward propagating field in (20), we have

$$
\sum_{\mathrm{j}} a_{\mathrm{j}} \mathbf{e}_{\mathrm{j}}^{+}\left(\mathbf{r}_{\perp}\right)=\sum_{\mathrm{j}, \mathrm{k}} c_{\mathrm{jk}}^{+} \alpha_{\mathrm{k}} \mathbf{e}_{\mathrm{j}}^{+}\left(\mathbf{r}_{\perp}\right),
$$

where $\bar{\alpha}=\left(\bar{c}^{+}\right)^{-1} \bar{a}$, whereas for the backward propagating field, we have

$$
\sum_{\mathrm{j}} b_{\mathrm{j}} \mathbf{e}_{\mathrm{j}}^{-}\left(\mathbf{r}_{\perp}\right)=\sum_{\mathrm{j}, \mathrm{k}} \gamma_{\mathrm{jk}}^{-} \alpha_{\mathrm{k}} \mathbf{e}_{\mathrm{j}}^{-}\left(\mathbf{r}_{\perp}\right),
$$

where $\overline{\bar{\gamma}}^{-}=\overline{\bar{P}}_{L / 2} \overline{\bar{R}}_{\mathrm{t}} \overline{\bar{P}}_{L / 2} \overline{\bar{c}}^{+}$. The total field can then be written as

$$
\mathbf{E}\left(\mathbf{r}_{\perp}, z_{0}\right)=\sum_{\mathrm{j}, \mathrm{k}} c_{\mathrm{jk}}^{+} \alpha_{\mathrm{k}} \mathbf{e}_{\mathrm{j}}^{+}\left(\mathbf{r}_{\perp}\right)+\sum_{\mathrm{j}, \mathrm{k}} \gamma_{\mathrm{jk}}^{-} \alpha_{\mathrm{k}} \mathbf{e}_{\mathrm{j}}^{-}\left(\mathbf{r}_{\perp}\right),
$$


and the cavity mode contribution is given by

$$
\mathbf{E}_{\mathrm{C}}\left(\mathbf{r}_{\perp}, z_{0}\right)=\sum_{\mathrm{j}} c_{\mathrm{jM}}^{+} \alpha_{\mathrm{M}} \mathbf{e}_{\mathrm{j}}^{+}\left(\mathbf{r}_{\perp}\right)+\sum_{\mathrm{j}} \gamma_{\mathrm{jM}}^{-} \alpha_{\mathrm{M}} \mathbf{e}_{\mathrm{j}}^{-}\left(\mathbf{r}_{\perp}\right) \text {. }
$$

\section{B. Far field calculation}

The electric field at the surface $z=z_{\mathrm{S}}$ of the micropillar is computed from the electric field expansion Eq. (20) using the total transmission matrix of the top DBR. We then determine the far field $\mathbf{E}_{\mathrm{F}}(\theta, \phi)$ on the surface of a sphere $S$ of radius $R$ using a standard near field to far field transformation ${ }^{54}$. To take into account the limited NA of the lens, we set $\mathbf{E}_{\mathrm{F}}\left(\theta>\theta_{\mathrm{Max}}, \phi\right)=0$ with $\theta_{\text {Max }}=\sin ^{-1}(\mathrm{NA})$. Following the procedure in Refs. 12 and 13, we then consider a Gaussian beam in the paraxial limit in a focal plane $z=z_{\mathrm{F}}$ given by

$$
\begin{aligned}
\mathbf{E}^{\mathrm{g}}\left(x, y, z_{\mathrm{F}}\right) & =\sqrt{\frac{2 c \mu_{0}}{\pi \mathrm{w}_{0}^{2}}} \exp \left(-\frac{x^{2}+y^{2}}{\mathrm{w}_{0}^{2}}\right) \hat{\mathbf{x}}, \\
\mathbf{H}^{\mathrm{g}}\left(x, y, z_{\mathrm{F}}\right) & =\frac{1}{\sqrt{c \mu_{0}}} \hat{\mathbf{z}} \times \mathbf{E}^{\mathrm{g}},
\end{aligned}
$$

where $\mathrm{w}_{0}$ is the beam waist and the integrated power in the plane $z=z_{\mathrm{F}}$ is normalized to 1 . We then compute the associated far field $\mathbf{E}_{\mathrm{F}}^{\mathrm{g}}$ and $\mathbf{H}_{\mathrm{F}}^{\mathrm{g}}$ on the surface $S$, and the overlap with the Gaussian beam is then given as

$$
O\left(\mathrm{w}_{0}, z_{\mathrm{F}}\right)=R^{2} \int\left(E_{\mathrm{F}, \theta}^{*} H_{\mathrm{F}, \phi}^{g}-E_{\mathrm{F}, \phi}^{*} H_{\mathrm{F}, \theta}^{g}\right) \sin \theta d \theta d \phi,
$$

where the subindices $\theta$ and $\phi$ indicate the field components in spherical coordinates. Finally, the transmission to the lens taking into account the coupling to the Gaussian profile is given by

$$
P_{\text {Lens }}=\frac{1}{2} \operatorname{Max}\left|O\left(\mathrm{w}_{0}, z_{\mathrm{F}}\right)\right|^{2} .
$$

The power $P_{\text {Lens,C }}$ coupled to the lens from the cavity is obtained by calculating $\mathbf{E}_{\mathrm{F}}(\theta, \phi)$ using the cavity contribution to the electric field given by Eq. (25) instead.

\section{Comparison of efficiency and the one from simplified model}

We compare the calculated efficiency $\varepsilon$ obtained from the full model Eq. (1) and the efficiency $\varepsilon_{s}$ from the single-mode model in Fig. 6. Excellent agreement between the two models is observed, indicating that the contribution from radiation modes to the collection of light in a Gaussian mode at the first lens is negligible.

\section{Modeling of sidewall roughness}

The sidewall scattering $Q_{\text {edge }}^{-1}$ can be computed using a model ${ }^{44}$ taking into account the ratio of the mode in-

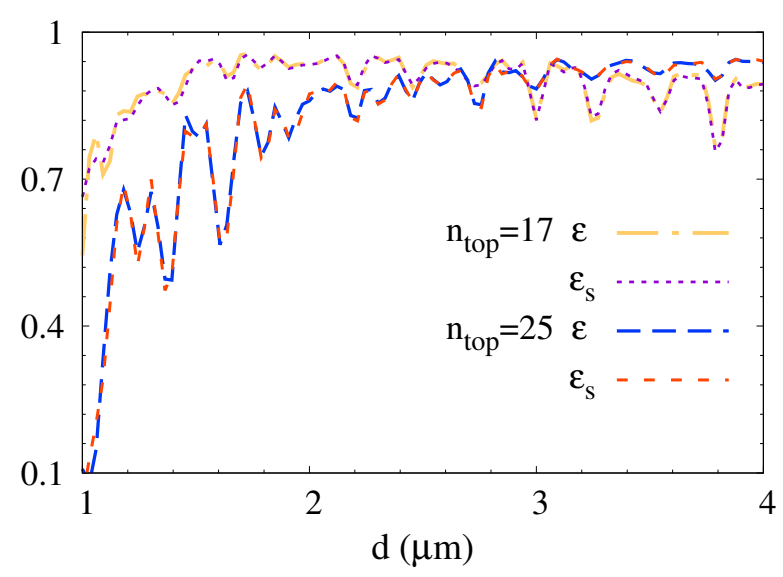

FIG. 6. The full efficiency $\varepsilon$ and the single-mode efficiency $\varepsilon_{s}$ computed using $n_{\text {top }}=17$ and 25 top DBR layer pairs. The ideal structure with $\kappa=0$ is considered.

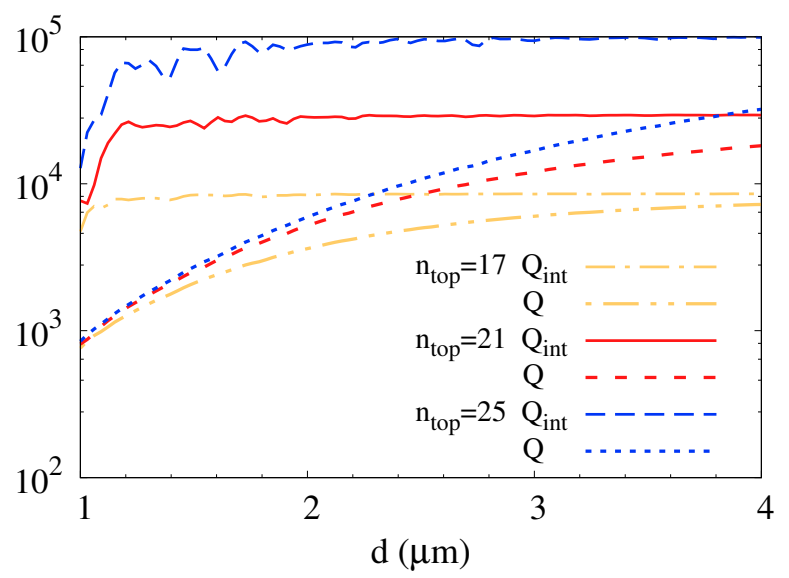

FIG. 7. The intrinsic cavity quality factor $Q_{\text {int }}$ and the $Q$ factor taking into account sidewall scattering as a function of pillar diameter $d$ for $\kappa=0.2 \mu \mathrm{m}$.

tensity at the micropillar edge and its diameter $d$ as

$$
Q_{\text {edge }}^{-1}=\kappa \frac{J_{0}^{2}\left(k_{\mathrm{t}} d / 2\right)}{d / 2} .
$$

Here, $\kappa$ is a phenomenological constant, $J_{0}$ is the Bessel function of the first kind, $k_{\mathrm{t}}$ is defined as $k_{\mathrm{t}}=\sqrt{n^{2} k_{0}^{2}-\beta_{1}^{2}}$ with $n$ being the refractive index of GaAs, $k_{0}=\omega / c$ and $\beta_{1}$ denoting the propagating constant of the fundamental $\mathrm{HE}_{11}$ mode in the cavity section. The $Q$ factor in the presence of sidewall roughness is then determined from Eq. (30) and the intrinsic quality factor $Q_{\text {int }}$ using Eq. (19) and is presented in Fig. 7. The sidewall roughness represents an additional decay channel of the light in the cavity leading to a reduction of $Q$, which becomes more significant for high intrinsic quality factors. As the pillar diameter increases, this influence decreases as the relative overlap of the $\mathrm{HE}_{11}$ mode with the boundary is reduced. 


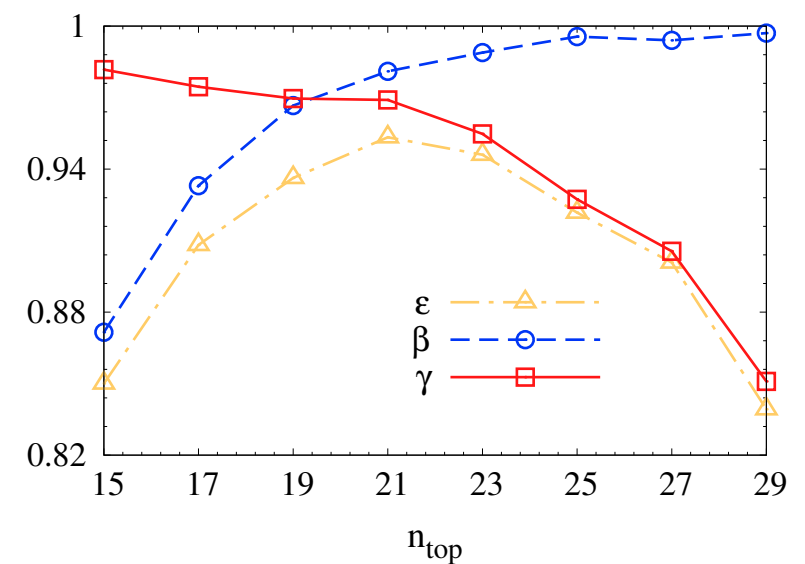

FIG. 8. The efficiency $\varepsilon$, the spontaneous emission $\beta$ factor and the transmission $\gamma$ as a function of top DBR layer pairs $n_{\text {top }}$ for a pillar diameter $d=3.2 \mu \mathrm{m}$ with $\kappa=0$.

\section{E. Dependence on the top DBR thickness}

The efficiency, the spontaneous emission $\beta$ factor and the transmission $\gamma$ are presented in Fig. 8 as function of the number of top DBR layer pairs. As $n_{\text {top }}$ increases, the cavity $Q$ factor and in turn the Purcell factor are improved leading to an increasing $\beta$. However, as the top mirror reflectivity becomes comparable to that of the bottom DBR, an increasing fraction of light penetrates into the substrate reducing the transmission $\gamma$. We thus have a trade-off between $\beta$ and $\gamma$, where an optimum efficiency of $\varepsilon \sim 0.95$ is obtained for $n_{\text {top }} \sim 21$. *ngre@fotonik.dtu.dk

1 J. L. O'Brien, A. Furusawa, and J. Vučković, Nat. Photonics 3, 687 (2009).

2 J. W. Pan, Z. B. Chen, C. Y. Lu, H. Weinfurter, A. Zeilinger, and M. Zukowski, Rev. Mod. Phys. 84, 777 (2012).

3 N. Gregersen, D. P. S. McCutcheon, and J. Mørk, in Handbook of Optoelectronic Device Modeling and Simulation Vol. 2, edited by J. Piprek (CRC Press, Boca Raton, 2017) Chap. 46, pp. 585-607.

4 N. Gregersen, P. Kaer, and J. Mørk, IEEE J. Sel. Top. Quantum Electron. 19, 9000516 (2013).

${ }^{5}$ H. Wang, J. Qin, X. Ding, M.-C. Chen, S. Chen, X. You, Y.-M. He, X. Jiang, L. You, Z. Wang, C. Schneider, J. J. Renema, S. Höfling, C.-Y. Lu, and J.-W. Pan, Phys. Rev. Lett. 123, 250503 (2019).

6 J. Renema, V. Shchesnovich, and R. Garcia-Patron, arXiv:1809.01953.

7 P. G. Kwiat, K. Mattle, H. Weinfurter, A. Zeilinger, A. V. Sergienko, and Y. Shih, Phys. Rev. Lett. 75, 4337 (1995).

8 A. J. Shields, Nat. Photonics 1, 215 (2007).

${ }^{9}$ H. Wang, H. Hu, T. H. Chung, J. Qin, X. Yang, J. P. Li, R. Z. Liu, H. S. Zhong, Y. M. He, X. Ding, Y. H. Deng, Q. Dai, Y. H. Huo, S. Höfling, C. Y. Lu, and J. W. Pan, Phys. Rev. Lett. 122, 113602 (2019).

10 J. Claudon, J. Bleuse, N. S. Malik, M. Bazin, P. Jaffrennou, N. Gregersen, C. Sauvan, P. Lalanne, and J. M. Gérard, Nat. Photonics 4, 174 (2010).

11 M. E. Reimer, G. Bulgarini, N. Akopian, M. Hocevar, M. B. Bavinck, M. A. Verheijen, E. P. Bakkers, L. P. Kouwenhoven, and V. Zwiller, Nat. Commun. 3, 737 (2012).

12 M. Munsch, N. S. Malik, E. Dupuy, A. Delga, J. Bleuse, J. M. Gérard, J. Claudon, N. Gregersen, and J. Mørk, Phys. Rev. Lett. 110, 177402 (2013).

13 M. Munsch, N. S. Malik, E. Dupuy, A. Delga, J. Bleuse, J. M. Gérard, J. Claudon, N. Gregersen, and J. Mørk, Phys. Rev. Lett. 111, 239902 (2013).
14 S. Ates, L. Sapienza, M. Davanco, A. Badolato, and K. Srinivasan, IEEE J. Sel. Top. Quantum Electron. 18, 1711 (2012).

15 L. Sapienza, M. Davanço, A. Badolato, and K. Srinivasan, Nat. Commun. 6, 7833 (2015).

16 J. Liu, R. Su, Y. Wei, B. Yao, S. F. C. da Silva, Y. Yu, J. Iles-Smith, K. Srinivasan, A. Rastelli, J. Li, and X. Wang, Nat. Nanotechnol. 14, 586 (2019).

17 H. Wang, Y. M. He, T. H. Chung, H. Hu, Y. Yu, S. Chen, X. Ding, M. C. Chen, J. Qin, X. Yang, R. Z. Liu, Z. C. Duan, J. P. Li, S. Gerhardt, K. Winkler, J. Jurkat, L. J. Wang, N. Gregersen, Y. H. Huo, Q. Dai, S. Yu, S. Höfling, C. Y. Lu, and J. W. Pan, Nat. Photonics 13, 770 (2019).

18 A. Berthelot, I. Favero, G. Cassabois, C. Voisin, C. Delalande, P. Roussignol, R. Ferreira, and J.-M. Gérard, Nat. Phys. 2, 759 (2006).

19 S. Reitzenstein and A. Forchel, J. Phys. D: Appl. Phys. 43, 033001 (2010).

20 X. Ding, Y. He, Z. C. Duan, N. Gregersen, M. C. Chen, S. Unsleber, S. Maier, C. Schneider, M. Kamp, S. Höfling, C. Y. Lu, and J. W. Pan, Phys. Rev. Lett. 116, 020401 (2016).

21 N. Somaschi, V. Giesz, L. De Santis, J. C. Loredo, M. P. Almeida, G. Hornecker, S. L. Portalupi, T. Grange, C. Antón, J. Demory, C. Gómez, I. Sagnes, N. D. Lanzillotti-Kimura, A. Lemaítre, A. Auffeves, A. G. White, L. Lanco, and P. Senellart, Nat. Photonics 10, 340 (2016).

${ }^{22}$ W. L. Barnes, G. Björk, J. M. Gérard, P. Jonsson, J. A. Wasey, P. T. Worthing, and V. Zwiller, Eur. Phys. J. D 18, 197 (2002).

23 A. Dousse, L. Lanco, J. Suffczyński, E. Semenova, A. Miard, A. Lemaître, I. Sagnes, C. Roblin, J. Bloch, and P. Senellart, Phys. Rev. Lett. 101, 267404 (2008).

24 S. Ates, S. M. Ulrich, S. Reitzenstein, A. Löffler, A. Forchel, and P. Michler, Phys. Rev. Lett. 103, 167402 (2009).

25 Y. M. He, H. Wang, C. Wang, M. C. Chen, X. Ding, J. Qin, Z. C. Duan, S. Chen, J. P. Li, R. Z. Liu, C. Schneider, M. Atatüre, S. Höfling, C. Y. Lu, and J. W. Pan, Nat. 
Phys. 15, 941 (2019).

26 S. E. Thomas, M. Billard, N. Coste, S. C. Wein, Priya, H. Ollivier, O. Krebs, L. Tazaïrt, A. Harouri, A. Lemaitre, I. Sagnes, C. Anton, L. Lanco, N. Somaschi, J. C. Loredo, and P. Senellart, arXiv:2007.04330.

27 J. Houel, A. V. Kuhlmann, L. Greuter, F. Xue, M. Poggio, B. D. Gerardot, P. A. Dalgarno, A. Badolato, P. M. Petroff, A. Ludwig, D. Reuter, A. D. Wieck, and R. J. Warburton, Phys. Rev. Lett. 108, 107401 (2012).

28 P. Kaer, N. Gregersen, and J. Mørk, New J. Phys. 15, 035027 (2013).

${ }^{29}$ K. Roy-Choudhury and S. Hughes, Phys. Rev. B 92, 205406 (2015).

30 E. V. Denning, M. Bundgaard-Nielsen, and J. Mørk, arXiv:2007.14719.

31 J. Iles-Smith, D. P. McCutcheon, A. Nazir, and J. Mørk, Nat. Photonics 11, 521 (2017).

32 E. V. Denning, J. Iles-Smith, A. D. Osterkryger, N. Gregersen, and J. Mork, Phys. Rev. B 98, 121306 (2018).

33 T. Häyrynen, J. R. de Lasson, and N. Gregersen, J. Opt. Soc. Am. A 33, 1298 (2016).

34 A. Lavrinenko, J. Lægsgaard, N. Gregersen, F. Schmidt, and T. Søndergaard, Numerical Methods in Photonics (CRC Press, 2014).

35 S. Gehrsitz, F. K. Reinhart, C. Gourgon, N. Herres, A. Vonlanthen, and H. Sigg, J. Appl. Phys. 87, 7825 (2000).

36 P. Lalanne, J. P. Hugonin, and J. M. Gérard, Appl. Phys. Lett. 84, 4726 (2004).

37 L. Novotny and B. Hecht, Principles of nano-optics (Cambridge university press, 2012).

${ }^{38}$ I. Wilson-Rae and A. Imamoğlu, Phys. Rev. B 65, 235311 (2002).

39 K. Roy-Choudhury and S. Hughes, Phys. Rev. B 92, 205406 (2015).

40 A. Nazir and D. P. McCutcheon, Journal of Physics: Condensed Matter 28, 103002 (2016).
41 E. V. Denning, J. Iles-Smith, N. Gregersen, and J. Mørk, Opt. Mater. Express 10, 222 (2020).

42 G. Lecamp, J. P. Hugonin, P. Lalanne, R. Braive, S. Varoutsis, S. Laurent, A. Lemaître, I. Sagnes, G. Patriarche, I. Robert-Philip, and I. Abram, Appl. Phys. Lett. 90, 091120 (2007).

43 N. Gregersen, S. Reitzenstein, C. Kistner, M. Strauss, C. Schneider, S. Höfling, L. Worschech, A. Forchel, T. R. Nielsen, J. Mørk, and J. M. Gérard, IEEE J. Quantum Electron. 46, 1470 (2010).

44 T. Rivera, J. P. Debray, J. M. Gérard, B. Legrand, L. Manin-Ferlazzo, and J. L. Oudar, Appl. Phys. Lett. 74, 911 (1999).

45 C. Schneider, P. Gold, S. Reitzenstein, S. Höfling, and M. Kamp, Appl. Phys. B 122, 19 (2016).

46 M. Karl, B. Kettner, S. Burger, F. Schmidt, H. Kalt, and M. Hetterich, Opt. Express 17, 1144 (2009).

47 S. Reitzenstein, N. Gregersen, C. Kistner, M. Strauss, C. Schneider, L. Pan, T. R. Nielsen, S. Höfling, J. Mørk, and A. Forchel, Appl. Phys. Lett. 94, 061108 (2009).

48 N. Gregersen, T. R. Nielsen, J. Claudon, J.-M. Gérard, and J. Mørk, Opt. Lett. 33, 1693 (2008).

49 A. Greilich, R. Oulton, E. A. Zhukov, I. A. Yugova, D. R. Yakovlev, M. Bayer, A. Shabaev, A. L. Efros, I. A. Merkulov, V. Stavarache, D. Reuter, and A. Wieck, Phys. Rev. Lett. 96, 227401 (2006).

50 M. Lermer, N. Gregersen, F. Dunzer, S. Reitzenstein, S. Höfling, J. Mørk, L. Worschech, M. Kamp, and A. Forchel, Phys. Rev. Lett. 108, 057402 (2012).

51 N. Gregersen, D. P. S. McCutcheon, J. Mørk, J.-M. Gérard, and J. Claudon, Opt. Express 24, 20904 (2016).

52 A. D. Osterkryger, J.-M. Gérard, J. Claudon, and N. Gregersen, Opt. Lett. 44, 2617 (2019).

53 J. Bleuse, J. Claudon, M. Creasey, N. S. Malik, J. M. Gérard, I. Maksymov, J. P. Hugonin, and P. Lalanne, Phys. Rev. Lett. 106, 103601 (2011).

54 C. A. Balanis, Advanced Engineering Electromagnetics, 1st ed. (Wiley, 1989). 